\title{
Modeling Longitudinal Gambling Data: Challenges and Opportunities
}

\author{
Kristoffer Magnusson \\ Centre for Psychiatry Research, Department of Clinical Neuroscience, \\ Karolinska Institutet \\ Anders Nilsson \\ Centre for Psychiatry Research, Department of Clinical Neuroscience, \\ Karolinska Institutet \\ Per Carlbring \\ Department of Psychology, \\ Stockholm University \\ 2019-09-11
}

\begin{abstract}
Clinical studies investigating treatments for problem gambling or gambling disorder frequently use gambling expenditure, such as gambling losses, as a treatment outcome. Gambling losses frequently vary substantially between participants; some report no losses, and some report substantial losses. In this article, we review how gambling losses are commonly analyzed in treatment studies, and show that frequently used methods, such as a $\log (y+1)$ transformation or assuming a normal distribution, often perform poorly for these types of data. We propose that a marginalized longitudinal two-part model is a more attractive option. The models are compared using real data from a trial including 136 persons with gambling disorder. Additionally, different performance metrics are further evaluated in a Monte Carlo simulation study. We conclude that gambling researchers should consider using the longitudinal two-part model as it offers a flexible and powerful way of modeling gambling outcomes. The $\log (y+1)$ transformation can be highly misleading in typical gambling data, as a difference in the number of zeros leads to biased estimates of the treatment effects.
\end{abstract}

Keywords: semicontinuous data, two-part models, longitudinal gambling data, power analysis

Gambling disorder is recognized as an addictive disorder (Petry, Blanco, Stinchfield,

\footnotetext{
Correspondence concerning this article should be sent to Kristoffer Magnusson, Centrum för psykiatriforskning, Norra Stationsgatan 69, 11364 Stockholm, Sweden. E-mail: kristoffer.magnusson@ki.se
} 
\& Volberg, 2013; Rennert et al., 2014), with severe consequences for the affected and their significant others (Langham et al., 2016). Efficacious psychological treatments exist, and cognitive behavior therapy (CBT) has the best empirical support (Cowlishaw et al., 2012).

Intervention studies on gambling disorder frequently include some behavioral measure of gambling behavior. For instance, 13 of the 14 CBT studies included in a Cochrane review of psychological treatments for gambling disorder included a measure of financial losses (Cowlishaw et al., 2012). These reports can be self-reported using diary-type methods, such as the timeline follow-back (TLFB; Weinstock, Whelan, \& Meyers, 2004), or using singleitem scales, or be based on real player data, e.g., when a responsible gambling intervention is evaluated in collaboration with the industry (Auer \& Griffiths, 2016; Ivanova, Magnusson, \& Carlbring, 2019; Jonsson, Hodgins, Munck, \& Carlbring, 2019). Behavioral measures commonly focus on days gambled and net money lost, which are thought to capture the majority of negative consequences associated with excessive gambling (Walker et al., 2006, p. 505). For that reason, gambling losses at posttest was chosen as one of the primary outcomes in the Cochrane review of psychological treatments (Cowlishaw et al., 2012). Although highly relevant as a treatment outcome, these types of measures pose methodological challenges that have not received adequate attention in the gambling literature.

Unfortunately, the issue of missing data is generally a big concern in gambling intervention studies (Grant, Kim, \& Kuskowski, 2004; Hodgins, Stea, \& Grant, 2011; Westphal, 2008). In a review of psychological treatments Melville, Casey, and Kavanagh (2007), found that the dropout ranged from $14 \%$ to $50 \%$, with a median of $26 \%$. It has become more common for investigators to include repeated measures as a way to reduce bias caused by missing data, and to better understand the mechanisms behind the treatment effect. Including repeated observations during the treatment, such as weekly TLFB reports, can increase the likelihood that dropout is related to the observed data, compared to a pretest and posttest only design. A longitudinal model fit using maximum likelihood offer an efficient method for retrieving unbiased treatment effects under such a scenario, or to perform more informative sensitivity analyses (Schafer \& Graham, 2002).

In this article, we provide a review of how gambling losses in intervention studies are usually analyzed. Our aim with this paper is to highlight some of the most apparent issues with the standard approaches, as well as to illustrate the opportunities offered by more flexible models. We present a motivational example using data from a recent trial, and use Monte Carlo simulations to demonstrate the performance under different scenarios of assuming a normal distribution, doing a log-transformation, or using a two-part model.

We reviewed the published intervention studies to understand how financial gambling outcomes are commonly handled in these studies. We included all trials (psychological and pharmacological interventions) from two recent reviews (Rodda et al., 2018; SBU, 2016), and included additional investigations published between January 2016 and August 2018. Although some studies included outcomes such as "reduced gambling," we focused on outcomes that were aimed to measure any form of continuous gambling expenditure, such as net losses or amount of money wagered. 
(a) Untransformed

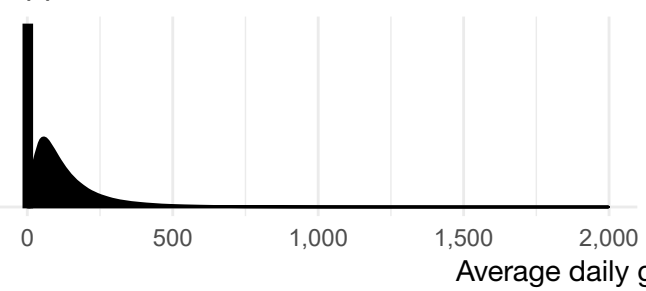

(b) $\log (y+1)$

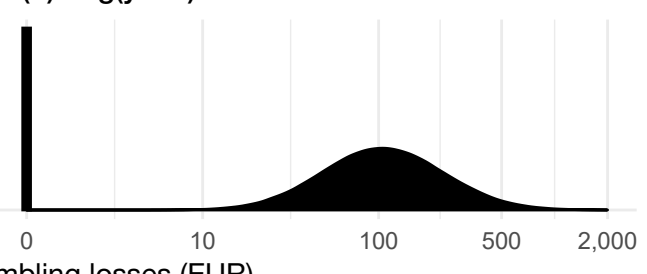

Figure 1. A $\log (y+1)$ transformation applied to lognormal distribution mixed with a number of zeros.

We reviewed 69 studies $^{1}$. Of those, $44(64 \%)$ included some type of gambling expenditure as an outcome. Among those 44 studies, the majority, $59 \%(n=26 / 44)$, noted that the outcome was heavily skewed. Among the 26 studies that discussed model assumptions, the majority chose to use a log-transformation $(54 \% ; n=14 / 26)$, some chose a non-parametric analysis $(15 \% ; n=4 / 26)$, and the rest mentioned only winsorizing their data or removing outliers.

\section{The Problem with the Log-transformation}

Most gambling data from treatment studies contain zeros-hopefully, quite a lot if the treatment is successful. However, none of the reviewed studies mentioned how they handled zeros. As it is well-known that the logarithm of 0 is undefined, we suspect that the authors used a shifted $\log$-transformation, i.e., $\log (y+c)$ where it is common to use $c=1$ to get rid of the zeros before doing the transformation. However, even in the case when the log-transformed losses (not including zeros) follow a normal distribution, including zeros causes the log-transformed outcome to no longer be normally distributed, as illustrated in Figure 1.

\section{Two-part Models}

As an alternative to assuming a normal response distribution (for the residual errors $)^{2}$ or transforming the outcome, we propose using a model with a more plausible datagenerating process. A parsimonious model that captures important features of addictive disorders is a two-part model (also known as hurdle or semicontinuous models). Two-part models have been proposed for the analysis of substance use disorder data by multiple authors (Atkins, Baldwin, Zheng, Gallop, \& Neighbors, 2013; Bandyopadhyay, DeSantis, Korte, \& Brady, 2011; DeSantis et al., 2013; Liu, Strawderman, Johnson, \& O'Quigley, 2016; Xing et al., 2015). Traditionally, two-part models (Olsen \& Schafer, 2001) combine a logistic model for whether a report will be zero or not zero, and model the actual losses or the number of drinks (the non-zero values) using a skewed continuous distribution, such as the lognormal or a gamma distribution, or a truncated Poisson or negative binomial distribution if it is a count outcome.

\footnotetext{
${ }^{1}$ Study authors KM and AN coded all studies independently

${ }^{2}$ When we write that we use or assume a normal distribution, we refer to the distribution of the residuals, or that $y_{i j}$ is assumed to follow a normal distribution. We are not saying that the actual data need to be normally distributed.
} 
Although the issues with zeros in gambling data share many similarities with the problem faced by researchers analyzing substance use data, there are some essential differences. The scale of the outcome differs substantially between gambling and substance use data. The number of, for instance, drinks per day or the number of cocaine use days has psychical limitations, whereas gambling losses can become extremely large within a very short period of time - making the distribution more extreme. Moreover, the consequences of losing, for instance, $€ 70,000$ in one day are not substantially different from spreading out the losses over seven days and losing $€ 10,000$ per day. The same cannot be said for substance use disorders, where there is a substantial difference between binge drinking seven drinks compared to having one drink per day for seven days. Consequently, as a treatment outcome, the overall reduction in gambling losses is more naturally the primary concern. It would be hard to argue that there is a beneficial treatment effect if there is a reduction in the number of days gambled while at the same time losses increase on the gambling days - so that the overall losses during the last 30 days have actually increased. Similarly, we would not claim that the treatment is beneficial if there is an increase in days gambled while there is a reduction in losses on days actually gambled; so that, again, the overall losses actually increase. Thus, the classical two-part model is not entirely satisfactory for gambling data as it leads to two separate treatment effects that can easily mislead. However, we recognize that in some scenarios, this is something desirable; for instance, when we want to learn about the change in abstinence, as well as the change in the intensity of the behavior when it occurs.

Instead of using the classical two-part model, we propose that a marginalized twopart model (Smith, Neelon, Preisser, \& Maciejewski, 2015) is a more attractive option for gambling data. A marginalized two-part model reformulates the classical two-part model so that the continuous part of the model refers to the overall losses (marginalized over the zeros and nonzero values), instead of modeling losses conditional on it being non-zero. Writing this as a two-level multilevel model leads to the following specification ${ }^{3}$,

Level 1 (Within-participants)

$$
\begin{aligned}
\operatorname{logit}\left(\pi_{i j}\right) & =\beta_{0 i}+\beta_{1 i} \text { Time }_{i j} & & \text { Zeros, } \\
\log \left(\nu_{i j}\right) & =\alpha_{0 i}+\alpha_{1 i} \text { Time }_{i j} & & \text { Losses. }
\end{aligned}
$$

Level 2 (Between-participants)

$$
\begin{array}{ll}
\beta_{0 i}=\gamma_{00}+\gamma_{01} \mathrm{TX}_{i}+U_{0 i} & \text { Zeros, Intercept } \\
\beta_{1 i}=\gamma_{10}+\gamma_{11} \mathrm{TX}_{i}+U_{1 i} & \text { Zeros, Slope } \\
\alpha_{0 i}=\delta_{00}+\delta_{01} \mathrm{TX}_{i}+U_{2 i} & \text { Losses, Intercept } \\
\alpha_{1 i}=\delta_{10}+\delta_{11} \mathrm{TX}_{i}+U_{3 i} & \text { Losses, Slope }
\end{array}
$$

where we have $i=1, \ldots, N$ subjects with $j=1, \ldots, n_{1 i}$ repeated measures per subject. $\mathrm{TX}_{i}$ denotes the participants' treatment allocation (0 or 1$)$, and $\mathrm{Time}_{i j}$ gives the value of the time variable. If we let $\mathrm{Time}_{i j}$ index weeks, and let the outcome be the average losses per day during each week, then $\pi_{i j}=\operatorname{Pr}\left(Y_{i j}=0 \mid U_{0 i}, U_{1 i}, \mathrm{TX}_{i}, \mathrm{Time}_{i j}\right)^{4}$ gives the

\footnotetext{
${ }^{3}$ We have left out the actual hurdle gamma density. Interested readers can find this in the Appendix

${ }^{4}$ In hurdle models $\pi$ is often used to refer to $\operatorname{Pr}(\mathrm{Y}>0)$, here we instead use $\operatorname{Pr}(\mathrm{Y}=0)$ in order to match
} 
probability of reporting no losses during the $j$ th week for participant $i$, and $\nu_{i j}=\mathrm{E}\left(Y_{i j} \mid\right.$ $U_{2 i}, U_{3 i}, \mathrm{TX}_{i}$, Time $\left._{i j}\right)$ gives the expected overall losses per day during the $j$ th week for participant $i$.

The model's random effects are assumed to be multivariate normal (on the link scale). Essentially, the two-part model for longitudinal data combines two generalized linear mixedeffects models (GLMM); i.e., the random effects are assumed to be,

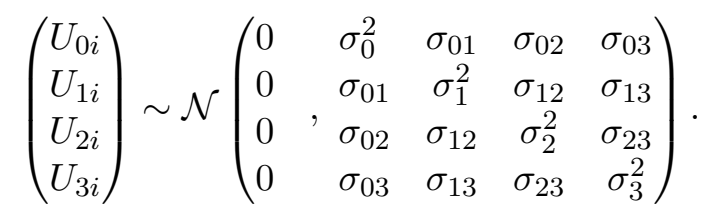

Thus, both parts of the model are linked via the correlated random effects. This means that we allow for a relationship between, for instance, having a higher likelihood of having zero losses at baseline and a change in the overall losses over time. A simplified version of the two-part model is illustrated in Figure 2.

\section{Interpreting the Treatment Effects}

As we use a logit link for the zero part and a log link for the overall losses, taking the exponent of both effects will give easily interpreted multiplicative effects. For the marginalized two-part gamma model given in Equation 1, the multiplicative effect for the overall losses at time point $t$ is given by,

$$
\frac{\mathrm{E}\left(Y_{i j} \mid \mathrm{TX}_{i}=1, \mathrm{Time}_{i j}=t, U_{2 i}, U_{3 i}\right)}{\mathrm{E}\left(Y_{i j} \mid \mathrm{TX}_{i}=0, \mathrm{Time}_{i j}=t, U_{2 i}, U_{3 i}\right)}=\exp \left(\delta_{11} \times t\right)
$$

Here $\delta_{11}$ is the parameter for the time $\times$ treatment-interaction for the overall losses. The multiplicative effect can then be easily interpreted as the percentages change in losses caused by the treatment at time point $t$, via $\% \Delta=\left(\exp \left(\delta_{11} \times t\right)-1\right) \times 100$. Similarly, $\exp \left(\gamma_{11} \times t\right)$ gives the subject-specific odds ratio for the treatment effect on reporting zero losses at time point $t$.

\section{Motivating Example: Individual Versus Couples Therapy}

To demonstrate how the study participants usually report their gambling losses, we use data from an randomized controlled trial (RCT) comparing two types of internetdelivered therapy for problem gamblers ( protocol$^{5}$ : Nilsson, Magnusson, Carlbring, Andersson, \& Hellner Gumpert, 2016). The trial included 136 gamblers and 136 concerned significant others, and randomized them to either individual CBT or a behavioral couples intervention. The participants filled out a TLFB report at baseline and weekly during 11 weeks of treatment. Figure 3 shows the posterior predictive distribution (Gelman, Meng, \& Stern, 1996) compared to the density of the real data. We can see that the predictions made using the marginalized two-part gamma model much better describe the observed data, compared to the $\log (y+1)$ transformation or the linear mixed-effects model (LMM).

how the model is parameterized in brms, the software we will use to fit the model.

${ }^{5}$ Main results have not been published 


\section{Level 2: Between-participants}

The distribution of participants' expected outcomes on the link scale

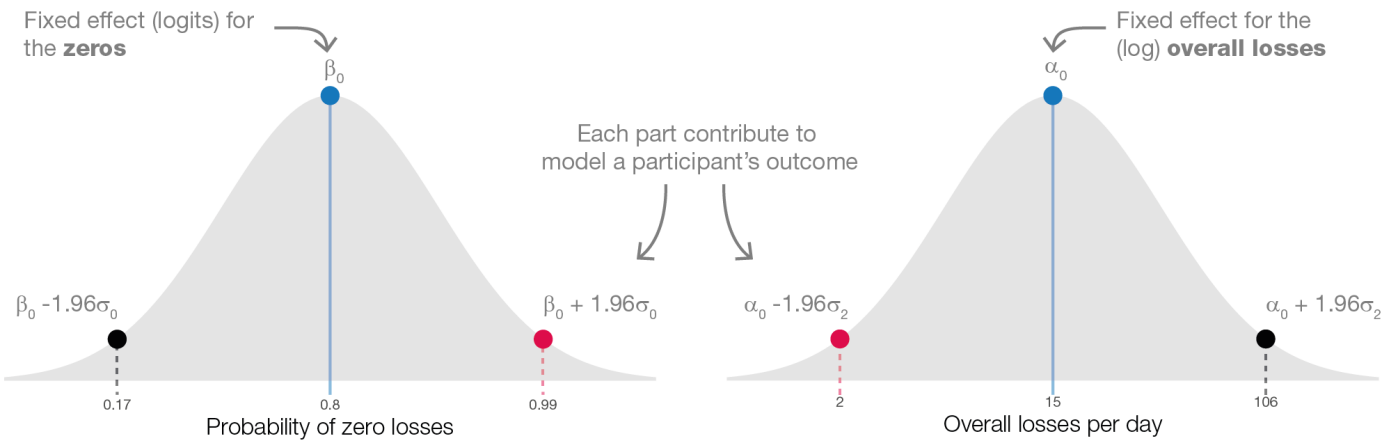

\section{Level 1: Within-participants}

The expected distribution of the outcome for the three different $(\bullet \bullet)$ combinations of random effects
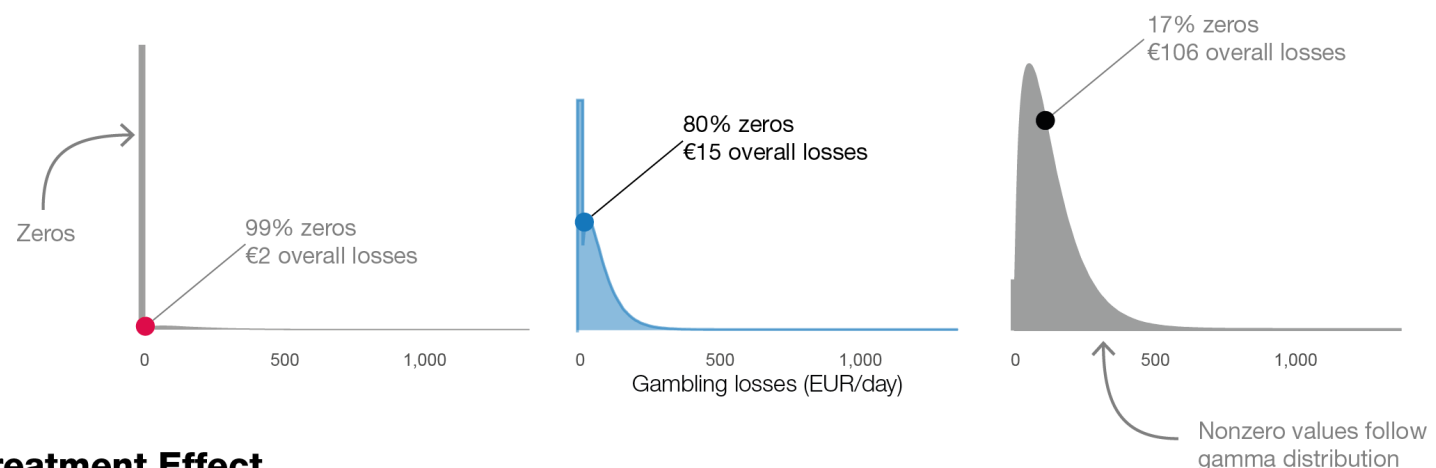

\section{Treatment Effect}

The multiplicative treatment effect shown on the original scale

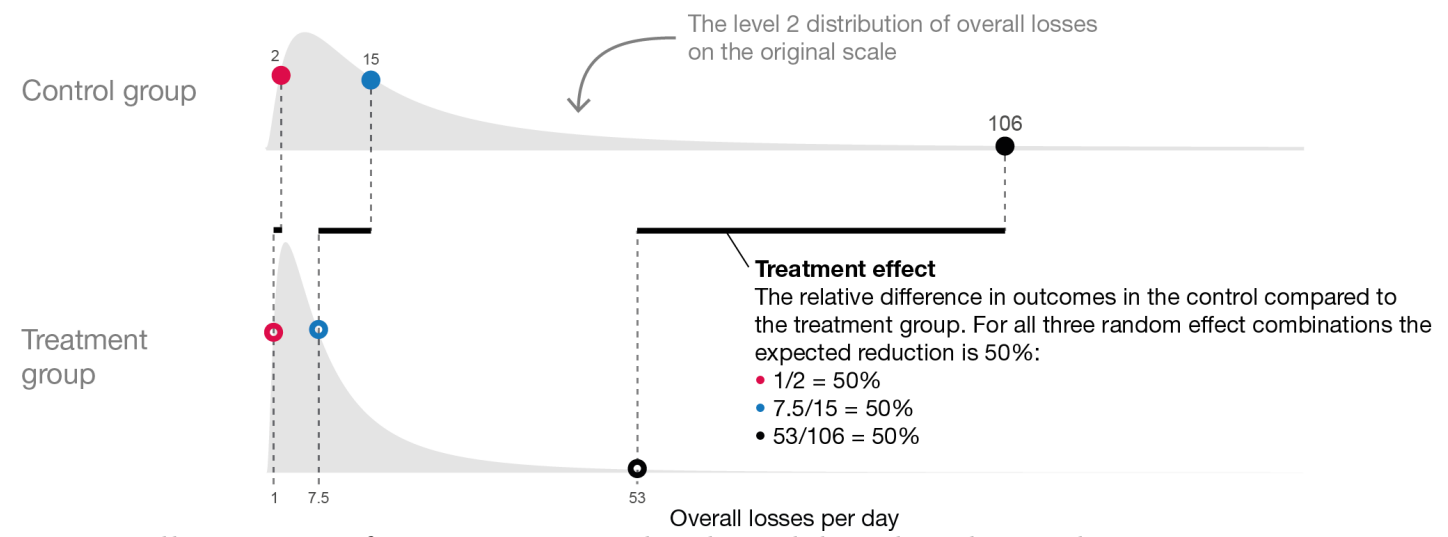

Figure 2. Illustration of a two-part two-level model with only random intercepts 
Table 1

Model Comparison Using 10-fold Cross-Validation

\begin{tabular}{lrc}
\hline Model & 10-fold IC & SE \\
\hline Two-part gamma & 3935.11 & 214.00 \\
Two-part LN & 3954.69 & 215.03 \\
LMM & 21337.03 & 4298.41 \\
LMM $(\log (y+1))$ & 6217.76 & 170.71 \\
Two-part gamma - Two-part LN & -19.58 & 5.25 \\
Two-part gamma - LMM & -17476.045 & 4263.19 \\
Two-part gamma - LMM $(\log (y+1))$ & -2282.65 & 53.04 \\
\hline
\end{tabular}

Note. 10 -fold IC $=-2 \times \widehat{e l p d}$, where elpd is the expected log pointwise predictive density; IC = information criteria; LN = lognormal; $\mathrm{SE}=$ standard error.

We also compared the models by comparing their predictive information criteria. This was performed by using 10-fold cross-validation (10-fold CV) to estimate each model's outof-sample predictive accuracy (Vehtari, Gelman, \& Gabry, 2017). The folds were stratified using the subject-level ID variable. Table 1 shows the comparisons, which indicate that the two-part model's predictive accuracy is substantially better that of the LMM and the LMM with a $\log (y+1)$ transformed outcome. However, any difference between the two-part gamma and the two-part lognormal model is small.

\section{Simulation Study}

The empirical example using real data showed that the LMM with an untransformed outcome and a $\log (y+1)$ transformed outcome failed to capture important features of the data. However, making bad predictions does not automatically mean that the two-part model will perform better on other metrics, such as bias and power. To further evaluate the models, a Monte Carlo simulation was performed. We designed five different simulation scenarios that were intended to highlight different types of issues with the models. The scenarios were as follows:

1. Scenario 1 (No effect): No effect of treatment on the overall losses or the proportion of zeros. The majority of the participants reduced their gambling when enrolled in the trial.

2. Scenario 2 (Control relapse): Same as Scenario 0 except that participants in the control group relapsed during the study period.

3. Scenario 3 (Control heavy gambling): In this scenario, participants in the control group continued to gamble quite heavily. In the treatment group, there was an immediate reduction in gambling when a participant entered treatment, as well as an improvement over time.

4. Scenario 4 (Difference in zeros): This scenario highlights the problem with the $\log (y+$ 1) transformation. There was no overall change in losses in the control group, but the proportion of the reports of no losses increased over time. In the treatment group, there was an overall reduction in losses, while at the same time, the proportion of 


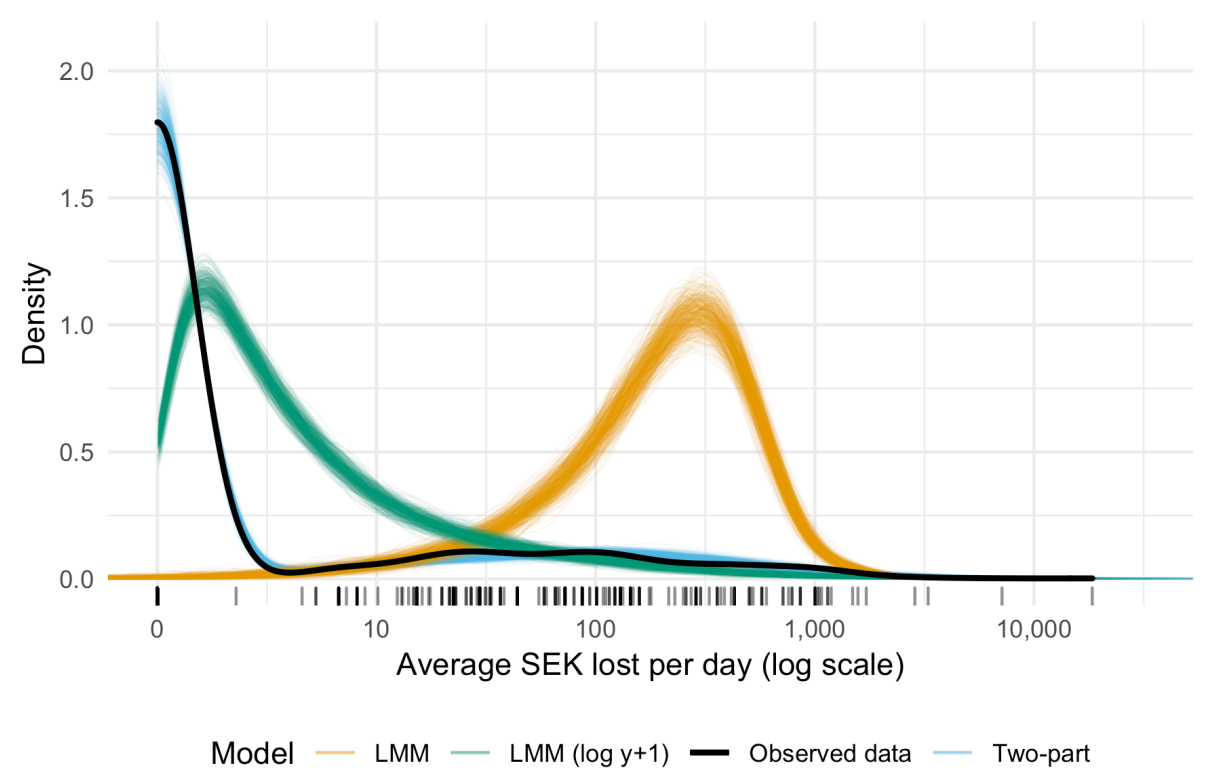

Figure 3. Posterior predictive distribution comparing the predictions from each model to the observed data. The rug over the $\mathrm{x}$-axis shows each observed data point. Values are in Swedish Krona (10.74 SEK = 1 EUR).

the reports of no losses decreased. This could happen if participants, for instance, reduced their gambling by gambling more moderately, perhaps by replacing a more problematic game with a less problematic game.

5. Scenario 5 (More variance): In this scenario, the variance between subjects was larger; in their odds of reporting zeros and overall losses. Most participants gambled quite lightly during the treatment period, while some gambled heavily. Thus, in the scenario, the difference between the mean and median losses was larger.

Figure 4 illustrates the different simulation scenarios, and Table 2 shows how the key parameters differ. We assumed that the reduction was linear on the log scale to avoid

Table 2

Simulation Setup

\begin{tabular}{|c|c|c|c|c|c|c|c|c|c|c|}
\hline \multirow[b]{2}{*}{ Scenario } & \multicolumn{4}{|c|}{ Intercept } & \multicolumn{2}{|c|}{ Post } & \multirow[b]{2}{*}{$\sigma_{0}^{2}$} & \multirow[b]{2}{*}{$\sigma_{1}^{2}$} & \multirow[b]{2}{*}{$\sigma_{2}^{2}$} & \multirow[b]{2}{*}{$\sigma_{3}^{2}$} \\
\hline & Losses (\$) & $\% \Delta$ & Zeros & OR & $\% \Delta$ & OR & & & & \\
\hline 1: No effect & 15 & 0 & $80 \%$ & 1 & 0 & 1 & 1.5 & 0.1 & 1 & 0.05 \\
\hline 2: Control relapse & 15 & 0 & $80 \%$ & 1 & -50 & 1.66 & 1.5 & 0.1 & 1 & 0.05 \\
\hline 3: Control heavy gambling & 100 & -50 & $20 \%$ & 3 & -75 & 4.5 & 1.5 & 0.1 & 1 & 0.05 \\
\hline 4: Diff. in zeros & 15 & 0 & $50 \%$ & 1 & -50 & 0.25 & 1.5 & 0.1 & 1 & 0.05 \\
\hline 5: More variance & 10 & 0 & $20 \%$ & 1 & -80 & 10 & 2.5 & 0.1 & 2 & 0.05 \\
\hline
\end{tabular}

Note. $\log (\operatorname{losses})=$ the intercept for overall losses; $\Delta \%$ gives the difference in intercepts; $\operatorname{logit}^{-1}$ (Zeros) $=$ the intercept for the zeros part; Intercept OR $=$ the odds ratio for the difference in intercepts for the zero part; Post $\Delta \%=$ the percentage difference 
making the simulation unnecessarily complicated. In all scenarios, the posttest occurred at week 10. We also varied the sample sizes at each level: at level 1 (repeated measures), $n_{1}=(3,6,11)$ and at level 2 (the number of subjects per treatment), $n_{2}=(25,50,75,150)$.

\section{Missing Data}

One of the reasons for repeatedly measuring participants during the treatment is that it can help reduce bias caused by treatment dropout. For that reasons, we included an additional simulation scenario where the likelihood of remaining in the study depended strongly on the reported value from the previous week, which interacted with the treatment assignment. Thus, the missing data mechanism fulfilled the missing at random (MAR) assumption. Participants in the treatment group who reported greater losses were more likely to drop out the next week, whereas participants on a waiting list (the control group) who lost more money were more likely to remain. In both groups, on average, around $35 \%$ of the participants were missing at the posttest.

To illustrate the apparent bias caused by the pretest-posttest design under these scenarios, we compared the LMMs and two-part models to a $t$-test of posttest scores, or an analysis of covariance (ANCOVA) where the pretest scores were included as a covariate.

\section{Assessment of the Simulations}

We evaluated the performance of the different models by calculating (for the estimates of the treatment effects at posttest) the coverage probabilities of the $95 \%$ confidence intervals (CIs), power, relative bias, and root mean squared error (RMSE). Treatment effects from the two-part model were estimated as differences on the log scale (the link scale), and the additive difference on the response scale. Effects on the response scale were calculated by marginalizing across the random effects using Monte Carlo integration (cf. Hedeker, du Toit, Demirtas, \& Gibbons, 2018) using all the posterior samples. The LMM with a $\log (y+1)$ transformed outcome was compared to the two-part model's effects on the log scale, and the classical LMM was compared to the additive effect on the response scale; thus, the two pairwise comparisons were on the same scale.

\section{Software and Implementation}

For each simulation, 2000 data sets were simulated, and each model was fit to each data set. All computations were performed using R version 3.5.1 (R Core Team, 2018), the two-part model was fit using Stan (Carpenter et al., 2017) using the brms package version 2.7.0 (Bürkner, 2017), the LMMs were fit using the package lme4 version 1.1-20 (Bates, Mächler, Bolker, \& Walker, 2015), and the simulations were performed using powerlmm version 0.5.0 (Magnusson, 2018).

The simulations were performed using resources provided by the Swedish National Infrastructure for Computing (SNIC) at the High-Performance Computing Center North (HPC2N), Umeå, Sweden.

An example of the code used to fit the model and run the simulation can be downloaded from the Open Science Framework: https://osf.io/6pbgv/. 


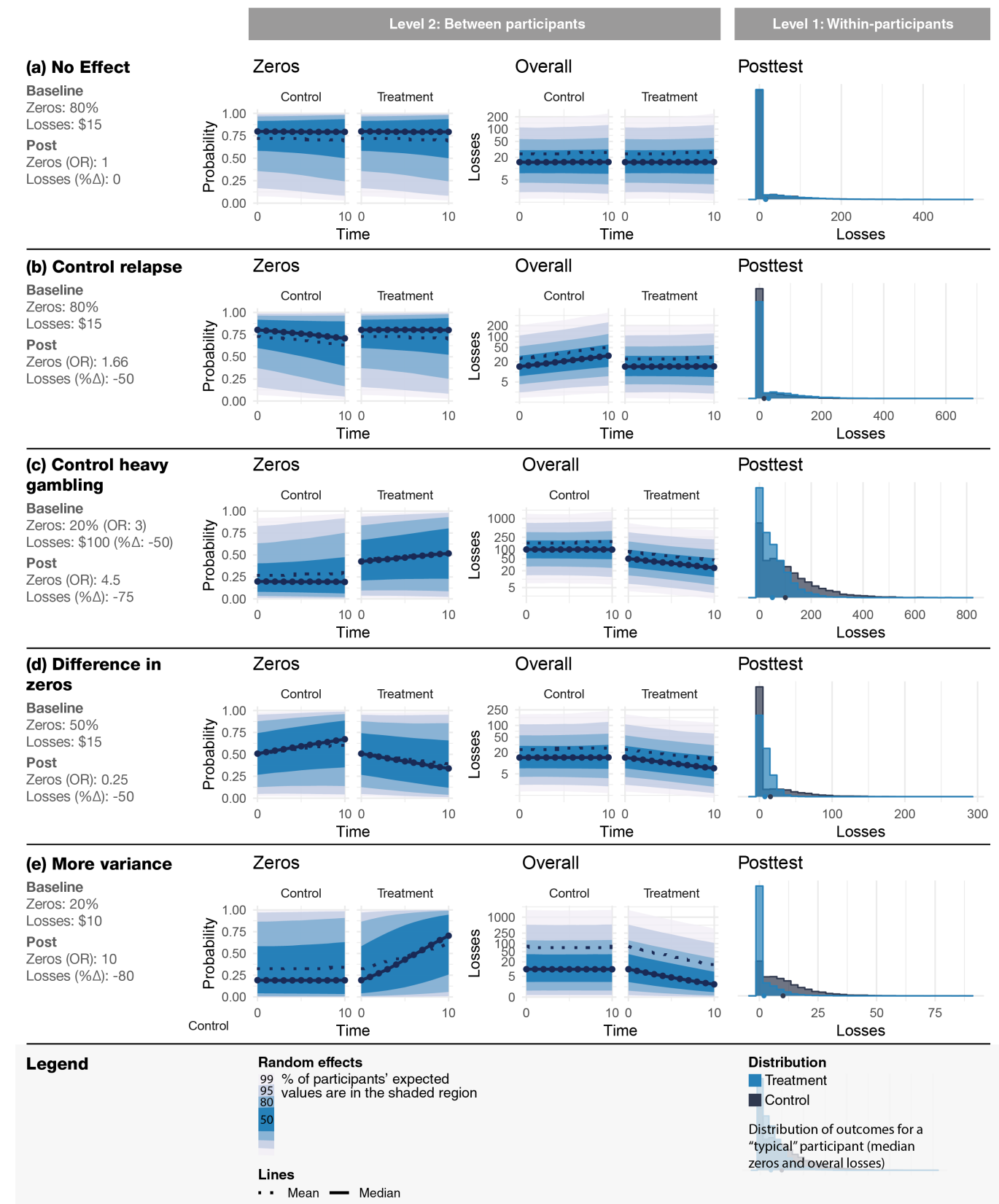

Figure 4. The five different simulation scenarios. The within-subjects plots show the expected distribution of losses for a subject with typical random effects at the posttest, i.e., at the center of each respective distribution. The dashed line shows the mean, and the solid line the median. The shaded regions show the $50 \%, 80 \%, 95 \%$, and $99 \%$ intervals of the expected values. 


\section{Simulation Results}

All models resulted in CIs that had acceptable coverage probabilities when there was no treatment effect, as shown in Figure 5. As expected, the LMM was quite robust, but the $95 \%$ CIs' coverage was negatively impacted in the two scenarios with more variance between subjects, and that larger sample sizes led to better coverage. The $\log (y+1)$ transformation's coverage was strongly impacted by the difference in the proportion of zeros, which led to worse results as the sample size increased. The two-part model had good coverage in all scenarios.

In all scenarios, the LMM yielded less power than the two-part model. The difference was most pronounced in model 4 (with more between-subject variance). The LMM had low sensitivity to even detect a $80 \%$ reduction in gambling losses. Figure 6 shows each model's power under the different scenarios. Power for the model with a $\log (y+1)$ transformation again depended on the difference in the proportion of zeros between treatment arms. In scenario 3 , the $\log (y+1)$ transformed model had close to $5 \%$ power for detecting a $50 \%$ reduction, whereas the two-part model had $80 \%$ power with 75 participants per group and 11 time points.

As expected, the LMMs estimated treatment effects with little bias, whereas the additive effects from the two-part model showed some bias for the models with few participants and few measurement points. For the log-scale coefficients, the two-part model showed little bias, whereas the $\log (y+1)$ transformed model was badly biased. Figure 7 shows the relative bias of the estimated treatment effect.

The estimated treatment effects from the LMMs generally deviated more for the true treatment effect compared to the two-part model, with the RMSE differing more as the between-subject variance increased. However, the RMSE for the $\log (y+1)$ transformed model was somewhat better than the two-part model in some scenarios, and substantially worse in others. Figure 8 shows the RMSE.

Table 3 shows results from the additional MAR missing data simulations. As was expected, using only complete data with a $t$-test or ANCOVA led to biased estimates of the treatment effect, which were very misleading in this scenario. The LMMs and the two-part model showed little to no bias, with close to nominal type I errors and CI coverage. Even with missing data, the two-part model was still more efficient than both LMMs.

\section{Discussion}

This paper focused on the challenges and the opportunities of modeling gambling losses. By reviewing the literature, we showed that the skewed nature of this type of outcome is well-recognized, and we gave several examples of why the two most popular methods for dealing with the model assumptions, a $\log (y+1)$ transformation or of using a normal response distribution are questionable choices. Instead, a marginalized two-part model that more closely matched our understanding of addictive disorders, such as gambling disorder, performed much better. The two-part model was shown to fit better using real data from a clinical trial, in terms of fit by predicting the observed data and in terms of out-of-sample predictive performance using 10-fold CV. Further problems with the logtransformation and the normal models were shown using simulations. We also argued that 
Table 3

MAR Missing Data Simulation Results

\begin{tabular}{|c|c|c|c|c|c|c|}
\hline Model & $n_{2}$ & CI cover & Type I error & Estimate & RMSE & $\mathrm{RB}(\mathrm{SE})$ \\
\hline \multicolumn{7}{|l|}{ Log scale } \\
\hline$t$-test $(\log (y+1))$ & $\begin{array}{r}25 \\
50 \\
75 \\
150\end{array}$ & $\begin{array}{l}0.78 \\
0.62 \\
0.47 \\
0.18\end{array}$ & $\begin{array}{l}0.22 \\
0.38 \\
0.53 \\
0.82\end{array}$ & $\begin{array}{l}-1.0 \\
-0.9 \\
-1.0 \\
-1.0\end{array}$ & $\begin{array}{l}1.3 \\
1.1 \\
1.1 \\
1.0\end{array}$ & $\begin{array}{l}0.01 \\
0.01 \\
0.00 \\
0.01\end{array}$ \\
\hline $\operatorname{ANCOVA}(\log (y+1))$ & $\begin{array}{r}25 \\
50 \\
75 \\
150\end{array}$ & $\begin{array}{l}0.84 \\
0.78 \\
0.67 \\
0.40\end{array}$ & $\begin{array}{l}0.16 \\
0.22 \\
0.33 \\
0.60\end{array}$ & $\begin{array}{l}-0.7 \\
-0.7 \\
-0.7 \\
-0.7\end{array}$ & $\begin{array}{l}1.1 \\
0.9 \\
0.9 \\
0.8\end{array}$ & $\begin{array}{c}0.00 \\
0.02 \\
-0.01 \\
0.01\end{array}$ \\
\hline $\operatorname{LMM}(\log (y+1))$ & $\begin{array}{r}25 \\
50 \\
75 \\
150\end{array}$ & $\begin{array}{l}0.94 \\
0.93 \\
0.94 \\
0.95\end{array}$ & $\begin{array}{l}0.06 \\
0.07 \\
0.06 \\
0.05\end{array}$ & $\begin{array}{l}0.0 \\
0.0 \\
0.0 \\
0.0\end{array}$ & $\begin{array}{l}0.6 \\
0.4 \\
0.4 \\
0.3\end{array}$ & $\begin{array}{l}-0.04 \\
-0.04 \\
-0.04 \\
-0.04\end{array}$ \\
\hline Two-part & $\begin{array}{r}25 \\
50 \\
75 \\
150\end{array}$ & $\begin{array}{l}0.95 \\
0.94 \\
0.95 \\
0.94\end{array}$ & $\begin{array}{l}0.05 \\
0.06 \\
0.05 \\
0.06\end{array}$ & $\begin{array}{l}0.0 \\
0.0 \\
0.0 \\
0.0\end{array}$ & $\begin{array}{l}0.4 \\
0.3 \\
0.2 \\
0.2\end{array}$ & $\begin{array}{c}0.05 \\
0.03 \\
0.01 \\
-0.01\end{array}$ \\
\hline Untransformed & & & & & & \\
\hline$t$-test & $\begin{array}{r}25 \\
50 \\
75 \\
150\end{array}$ & $\begin{array}{l}0.85 \\
0.72 \\
0.55 \\
0.27\end{array}$ & $\begin{array}{l}0.15 \\
0.28 \\
0.45 \\
0.73\end{array}$ & $\begin{array}{c}-93.6 \\
-97.4 \\
-104.7 \\
-106.9\end{array}$ & $\begin{array}{l}169.0 \\
136.1 \\
131.0 \\
118.7\end{array}$ & $\begin{array}{l}-0.19 \\
-0.11 \\
-0.08 \\
-0.05\end{array}$ \\
\hline ANCOVA & $\begin{array}{r}25 \\
50 \\
75 \\
150\end{array}$ & $\begin{array}{l}0.84 \\
0.72 \\
0.57 \\
0.27\end{array}$ & $\begin{array}{l}0.16 \\
0.28 \\
0.43 \\
0.73\end{array}$ & $\begin{array}{c}-96.0 \\
-97.2 \\
-103.4 \\
-105.3\end{array}$ & $\begin{array}{l}169.6 \\
136.9 \\
130.2 \\
118.3\end{array}$ & $\begin{array}{l}-0.20 \\
-0.11 \\
-0.07 \\
-0.04\end{array}$ \\
\hline LMM & $\begin{array}{r}25 \\
50 \\
75 \\
150\end{array}$ & $\begin{array}{l}0.95 \\
0.95 \\
0.95 \\
0.94\end{array}$ & $\begin{array}{l}0.05 \\
0.05 \\
0.05 \\
0.06\end{array}$ & $\begin{array}{c}-12.2 \\
-11.5 \\
-11.5 \\
-8.3\end{array}$ & $\begin{array}{c}146.7 \\
66.6 \\
55.2 \\
36.2\end{array}$ & $\begin{array}{l}-0.39 \\
-0.09 \\
-0.09 \\
-0.02\end{array}$ \\
\hline Two-part & $\begin{array}{r}25 \\
50 \\
75 \\
150\end{array}$ & $\begin{array}{l}0.95 \\
0.95 \\
0.95 \\
0.94\end{array}$ & $\begin{array}{l}0.05 \\
0.05 \\
0.05 \\
0.06\end{array}$ & $\begin{array}{c}1.0 \\
1.0 \\
-1.0 \\
-1.8\end{array}$ & $\begin{array}{l}77.7 \\
51.9 \\
42.0 \\
29.8\end{array}$ & $\begin{array}{l}0.58 \\
0.16 \\
0.10 \\
0.05\end{array}$ \\
\hline
\end{tabular}

Note. $n_{2}=$ the number of participants per group; CI coverage $=$ the proportion of $95 \%$ CIs that includes the true value; Type I error = the proportion of tests that falsely rejected the null hypothesis; Estimate = the mean of the estimates from each simulated data set; RMSE = root mean squared error; $\mathrm{RB}(\mathrm{SE})=$ Relative bias of the estimated standard error. 


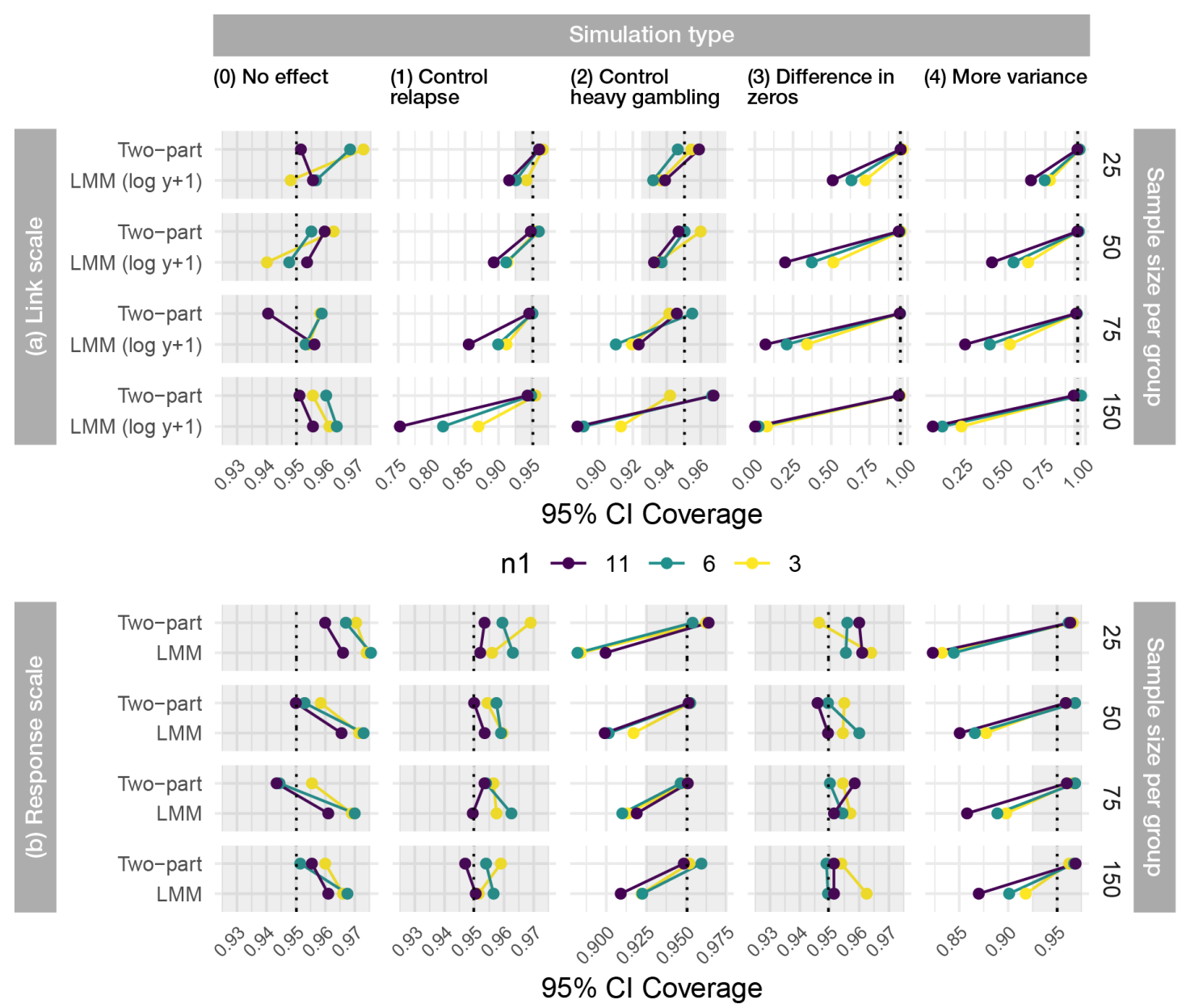

Figure 5. The empirical coverage for the treatment effects $95 \%$ CIs

as a treatment outcome, the overall reduction in gambling losses is a natural focus, which is provided by the marginalized two-part model.

As the simulations showed, it is hard to recommend the $\log (y+1)$ transformation unless one is reasonably certain that the proportion of zeros will not differ between groups; which would be a strange assumption to make in an RCT. Moreover, we saw that in many cases it would be better to leave the outcome untransformed, and use the classical LMM, which is more robust compared to the $\log (y+1)$ transformation. Clearly, if a treatment is successful, we expect to see a substantial difference in the number of zeros in the data. Except convenience, there is little reason to prefer the LMM over the two-part model. The two-part model not only generally performed better but also answered two essential questions: Does the treatment lead to any change in the likelihood of reporting no losses (a zero)? Does the treatment cause a change in the overall losses? Perhaps more importantly, treatment effects estimated using the LMM are additive treatment effect; i.e., they represent the mean difference between the treatment and the control. With possibly heavily skewed gambling data the mean difference can be considerably larger than the median, and thus, the estimated reduction in losses will not be relevant to the typical patient. Conversely, 


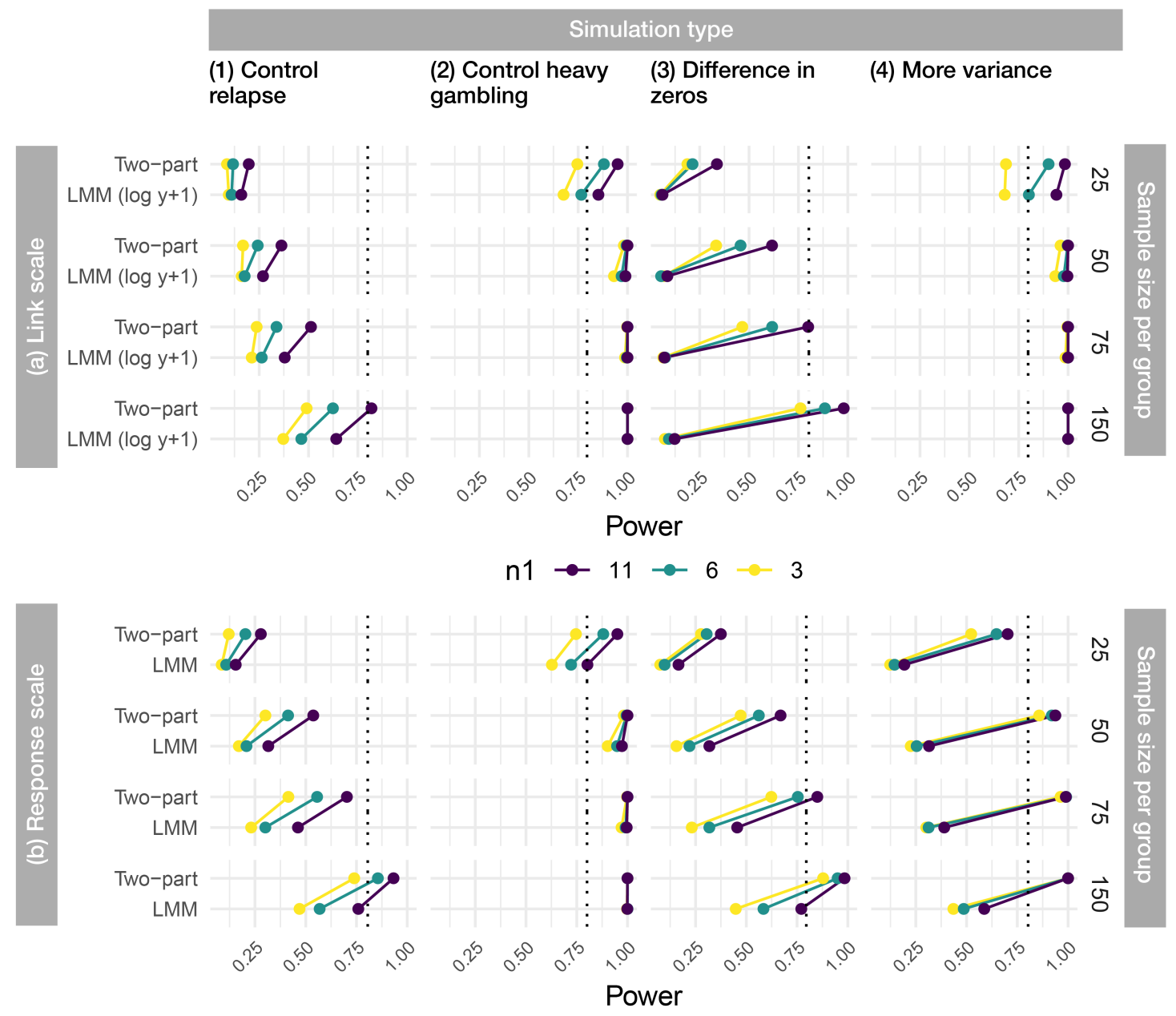

Figure 6. Empirical power from each simulation scenario

the two-part model with a log-link gives us the subject-specific effects as a ratio of the losses after being assigned to the treatment group compared to after being assigned to the control group. There is also the possibility of back-transforming the effects to the original money scale, and interpreting that effect at different percentiles. For instance, the backtransformed fixed effects can be interpreted as the predicted values for the "typical patient" (with their random intercept and slope at the center of the distribution).

As most investigators have experienced, missing data is to be expected in gambling trials. As the MAR simulation showed, including only pretest and posttest measures leads to biased results in scenarios where the longitudinal MAR-based model would recover the true effect. Naturally, we do not know whether a participant's decision to drop out is related to the observed data or unobserved data (i.e., participants drop out because of large losses that happened after the last recorded value). However, studies with repeated measures make the MAR assumption somewhat more plausible, while also enabling better options 


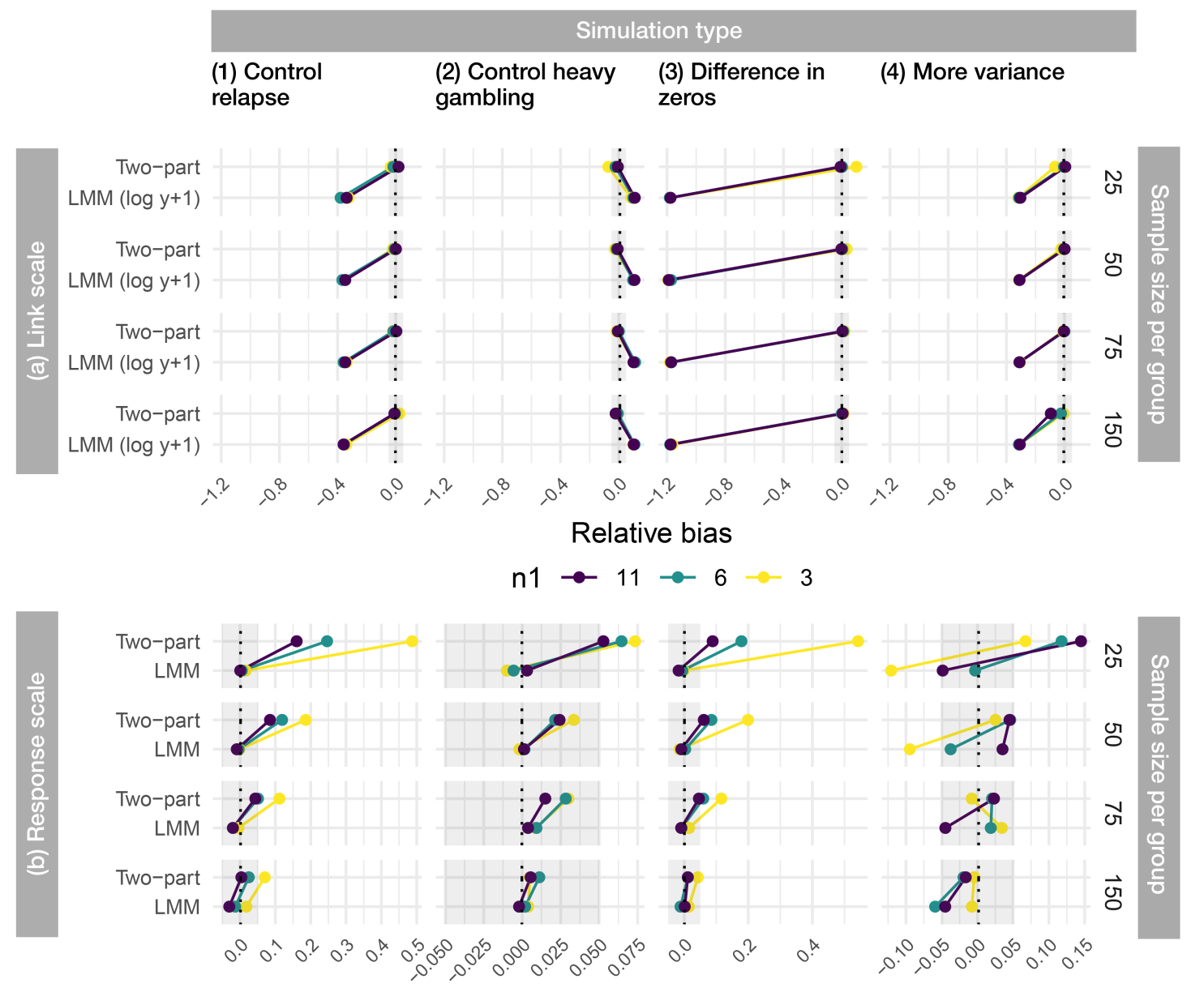

Relative bias

Figure 7. Relative bias for the treatment effects

for sensitivity analysis under different missing not at random (MNAR) scenarios, such as the pattern-mixture method (Little, 1993; Maruotti, 2011).

It is likely that we will see more reliable and detailed data on gambling expenditure that is longitudinal, either in future studies employing modern data collection techniques, such as ecological momentary assessment (Shiffman, Stone, \& Hufford, 2008), or using behavior tracking. Gambling research might be unique in the possibilities offered by behavioral tracking of gambling. It is possible for researchers to get access to ecologically valid data on a transactional level; however, investigations performed with real player data can be very heterogeneous with considerable differences in losses. Evaluation of, for example, responsible gambling tools offered by the operators, and the data generated, will require appropriate statistical methods to gain insight into the gambling behavior.

Evaluating gambling interventions is made harder by the large non-specific effects associated with even minimal-treatment approaches, and the powerful effect of deciding 


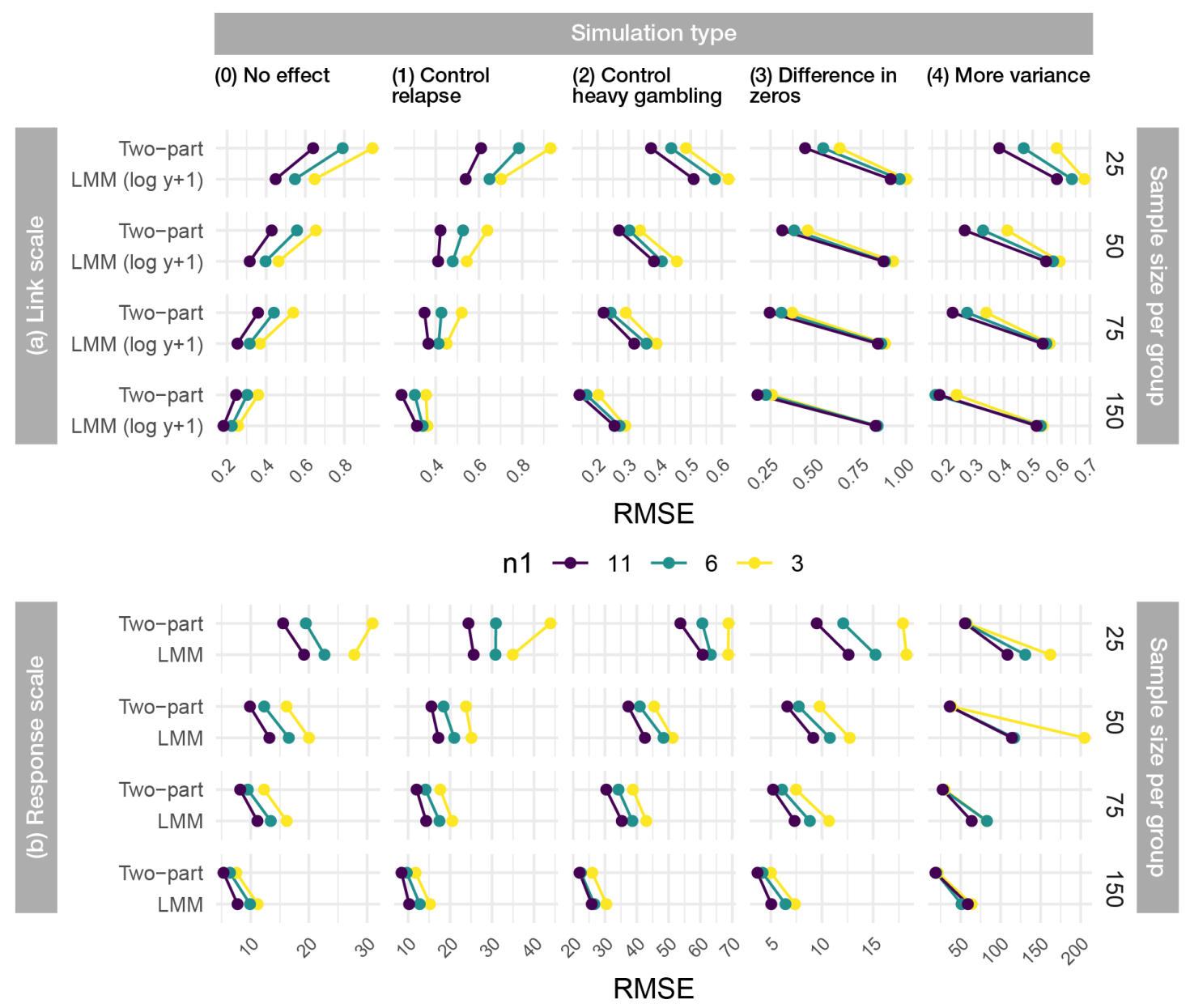

Figure 8. Root mean squared error (RMSE) for the treatment effects

to enter treatment (Diskin \& Hodgins, 2009; Toneatto \& Ladoceur, 2003), which leads to difficulty in detecting possible treatment effects. To increase the chances of detecting clinically meaningful effects, we need longitudinal follow-up measures and use statistical models that are more powerful. As the simulations showed, the two-part model has the potential to be more powerful and reliable in scenarios where the $\log (y+1)$ transformation and the classical LMM have low power or give very unreliable results.

The issues discussed in this article regarding gambling losses also likely apply to other gambling outcomes. Outcome scales such as the National Opinion Research Center Screen for Gambling Problems (NODS; Wickwire, Burke, Brown, Parker, \& May, 2008) or the Problem Gambling Severity Index (PGSI; Ferris \& Wynne, 2001) can also be influenced by the fact that some participants quit gambling and some gamble heavily. For instance, Martens, Arterberry, Takamatsu, Masters, and Dude (2015) analyzed the PGSI using a GLMM for that reason.

It is common to aggregate the reported losses, as we did in this report. However, if one wishes, one can include the daily losses nested within weekly or monthly reports. This is probably most relevant for studies using ecological momentary assessment in order 
to understand gambling behavior better, and it might be less attractive when evaluating treatment outcomes. However, from the point of view of missing data, important information might be lost when aggregating. For instance, after aggregating, the daily records for the last 7 days $(0,0,0,0,0,0,700)$ and $(100,100,100,100,100,100,100)$ will be the same. It is possible that such a loss of information is problematic if the likelihood of dropping out of the study is related to losing more money on a given day.

Marginalized two-part models can be fit using widely used software such as SAS NLMIXED (cf., Smith et al., 2015) or in R using Stan. However, the models are more complicated to fit, and investigators unfamiliar with them will probably need to consult an expert. An annotated example of how to fit the models in $\mathrm{R}$ is included as an online supplement ${ }^{6}$.

The marginalized two-part model considered in this paper can be extended in, at least, two important ways. First, in this paper, we compared a two-part model with either a gamma or a lognormal response distribution. More flexible options exist, such a threeparameter generalized gamma distribution, which includes the lognormal and the standard gamma distribution as special cases (Manning, Basu, \& Mullahy, 2005). Second, it is commonly assumed that gambling is influenced by past behavior, which is evident from one of the criteria for gambling disorder in the 5th edition of the Diagnostic and Statistical Manual of Mental Disorders (DSM-5): "After losing money gambling, often returns another day to get even ('chasing' ones losses)" (American Psychiatric Association, 2013, p. 585). Including an autoregressive component seems like a natural extension of the proposed twopart model. However, it is unclear whether these theoretical extensions would have an important impact when treatment effects are evaluated.

\section{Limitations}

The simulation examples in this paper had a clear "house advantage" as the two-part model used the correctly specified gamma distribution. Future studies should investigate the consequences of misspecifying the continuous response distribution or investigate the performance of a more flexible option such as the previously mentioned generalized gamma distribution in a gambling context. Moreover, most psychological treatments are delivered by therapists. Ignoring therapist effects in longitudinal data can have large consequences for type I errors (Magnusson, Andersson, \& Carlbring, 2018). We did not investigate how well the two-part model would perform if a third level (therapists) were included in the model. Last, we did not compare the classical two-part model to the marginalized two-part as we prefer the latter, because it naturally provides a more relevant estimate of treatment effects. However, it possible that the classical two-part model would have a better predictive performance, and that model should be considered if overall losses are not the primary interest.

\section{Conclusion}

Using an LMM with or without the $\log (y+1)$ transformation performs poorly compared to a two-part model. The $\log (y+1)$ transformation is especially bad when there is a difference in the proportion of reported zeros. The marginalized two-part model offers a

${ }^{6}$ Available for download from https://osf.io/6pbgv/ 
flexible and feasible way of analyzing gambling losses, yielding estimates of the treatment effects that are more efficient and relevant.

\section{References}

American Psychiatric Association. (2013). Diagnostic and statistical manual of mental disorders (DSM-5). Washington, DC: American Psychiatric Publishing.

Atkins, D. C., Baldwin, S. A., Zheng, C., Gallop, R. J., \& Neighbors, C. (2013). A tutorial on count regression and zero-altered count models for longitudinal substance use data. Psychology of Addictive Behaviors, 27(1), 166-177. doi:10.1037/a0029508

Auer, M. M. \& Griffiths, M. D. (2016). Personalized Behavioral Feedback for Online Gamblers: A Real World Empirical Study. Frontiers in Psychology, 7. doi:10.3389/fpsyg. 2016.01875. pmid: 27965611

Bandyopadhyay, D., DeSantis, S. M., Korte, J. E., \& Brady, K. T. (2011). Some Considerations for Excess Zeroes in Substance Abuse Research. The American Journal of Drug and Alcohol Abuse, 37(5), 376-382. doi:10.3109/00952990.2011.568080

Bates, D., Mächler, M., Bolker, B., \& Walker, S. (2015). Fitting linear mixed-effects models using lme4. Journal of Statistical Software, 67(1), 1-48. doi:10.18637/jss.v067.i01

Bürkner, P.-C. (2017). Brms: An R Package for Bayesian Multilevel Models Using Stan. Journal of Statistical Software, $80(1), 1-28$. doi:10.18637/jss.v080.i01

Carpenter, B., Gelman, A., Hoffman, M., Lee, D., Goodrich, B., Betancourt, M., ... Riddell, A. (2017). Stan: A probabilistic programming language. Journal of Statistical Software, Articles, 76 (1), 1-32. doi:10.18637/jss.v076.i01

Cowlishaw, S., Merkouris, S., Dowling, N., Anderson, C., Jackson, A., \& Thomas, S. (2012). Psychological therapies for pathological and problem gambling. The Cochrane Database of Systematic Reviews, 11, CD008937. doi:10.1002/14651858.CD008937. pub2. pmid: 23152266

DeSantis, S. M., Bandyopadhyay, D., Baker, N. L., Randall, P. K., Anton, R. F., \& Prisciandaro, J. J. (2013). Modeling longitudinal drinking data in clinical trials: An application to the COMBINE study. Drug and Alcohol Dependence, 132(1-2), 244-250. doi:10.1016/j.drugalcdep.2013.02.013. pmid: 23566774

Diskin, K. M. \& Hodgins, D. C. (2009). A randomized controlled trial of a single session motivational intervention for concerned gamblers. Behaviour Research and Therapy, 47(5), 382-388. doi:10.1016/j.brat.2009.01.018. pmid: 19249015

Ferris, J. A. \& Wynne, H. J. (2001). The Canadian problem gambling index. Canadian Centre on Substance Abuse Ottawa, ON.

Gelman, A., Meng, X.-L., \& Stern, H. (1996). Posterior predictive assessment of model fitness via realized discrepancies. Statistica Sinica, 6(4), 733-760. JSTOR: 24306036

Grant, J. E., Kim, S. W., \& Kuskowski, M. (2004). Retrospective review of treatment retention in pathological gambling. Comprehensive Psychiatry, 45(2), 83-87. doi:10. 1016/j.comppsych.2003.12.005. pmid: 14999657

Hedeker, D., du Toit, S. H. C., Demirtas, H., \& Gibbons, R. D. (2018). A note on marginalization of regression parameters from mixed models of binary outcomes. Biometrics, 74 (1), 354-361. doi:10.1111/biom.12707

Hodgins, D. C., Stea, J. N., \& Grant, J. E. (2011). Gambling disorders. The Lancet, 378 (9806), 1874-1884. doi:10.1016/S0140-6736(10)62185-X 
Ivanova, E., Magnusson, K., \& Carlbring, P. (2019). Deposit Limit Prompt in Online Gambling for Reducing Gambling Intensity: A Randomized Controlled Trial. Frontiers in Psychology, 10. doi:10.3389/fpsyg.2019.00639

Jonsson, J., Hodgins, D. C., Munck, I., \& Carlbring, P. (2019). Reaching out to big losers: A randomized controlled trial of brief motivational contact providing gambling expenditure feedback. Psychology of Addictive Behaviors, 33(3), 179-189. doi:10.1037/ adb0000447

Langham, E., Thorne, H., Browne, M., Donaldson, P., Rose, J., \& Rockloff, M. (2016). Understanding gambling related harm: A proposed definition, conceptual framework, and taxonomy of harms. BMC Public Health, 16(1), 80. doi:10.1186/s12889-016-27470

Little, R. J. A. (1993). Pattern-Mixture Models for Multivariate Incomplete Data. Journal of the American Statistical Association, 88 (421), 125-134. doi:10.1080/01621459.1993. 10594302

Liu, L., Strawderman, R. L., Johnson, B. A., \& O'Quigley, J. M. (2016). Analyzing repeated measures semi-continuous data, with application to an alcohol dependence study. Statistical Methods in Medical Research, 25(1), 133-152. doi:10.1177/0962280212443324. pmid: 22474003

Magnusson, K. (2018). Powerlmm: Power analysis for longitudinal multilevel models.

Magnusson, K., Andersson, G., \& Carlbring, P. (2018). The consequences of ignoring therapist effects in trials with longitudinal data: A simulation study. Journal of Consulting and Clinical Psychology, 86(9), 711-725. doi:10.1037/ccp0000333

Manning, W. G., Basu, A., \& Mullahy, J. (2005). Generalized modeling approaches to risk adjustment of skewed outcomes data. Journal of Health Economics, 24(3), 465-488. doi:10.1016/j.jhealeco.2004.09.011

Martens, M. P., Arterberry, B. J., Takamatsu, S. K., Masters, J., \& Dude, K. (2015). The efficacy of a personalized feedback-only intervention for at-risk college gamblers. Journal of Consulting and Clinical Psychology, 83(3), 494-499. doi:10.1037/a0038843

Maruotti, A. (2011). A two-part mixed-effects pattern-mixture model to handle zeroinflation and incompleteness in a longitudinal setting. Biometrical Journal, 53(5), 716-734. doi:10.1002/bimj.201000190

Melville, K. M., Casey, L. M., \& Kavanagh, D. J. (2007). Psychological treatment dropout among pathological gamblers. Clinical Psychology Review, 27(8), 944-958. doi:10 . 1016/j.cpr.2007.02.004. pmid: 17433853

Nilsson, A., Magnusson, K., Carlbring, P., Andersson, G., \& Hellner Gumpert, C. (2016). Effects of added involvement from concerned significant others in internet-delivered CBT treatments for problem gambling: Study protocol for a randomised controlled trial. BMJ open, 6 (9), e011974. doi:10.1136/bmjopen-2016-011974

Olsen, M. K. \& Schafer, J. L. (2001). A two-part random-effects model for semicontinuous longitudinal data. Journal of the American Statistical Association, 96(454), 730-745. doi:10.1198/016214501753168389

Petry, N. M., Blanco, C., Stinchfield, R., \& Volberg, R. (2013). An empirical evaluation of proposed changes for gambling diagnosis in the DSM-5. Addiction, 108(3), 575-581. doi:10.1111/j.1360-0443.2012.04087.x 
R Core Team. (2018). R: A language and environment for statistical computing. Vienna, Austria: R Foundation for Statistical Computing.

Rennert, L., Denis, C., Peer, K., Lynch, K. G., Gelernter, J., \& Kranzler, H. R. (2014). DSM5 Gambling Disorder: Prevalence and Characteristics in a Substance Use Disorder Sample. Experimental and clinical psychopharmacology, 22(1), 50-56. doi:10.1037/ a0034518. pmid: 24490711

Rodda, S., Merkouris, S. S., Abraham, C., Hodgins, D. C., Cowlishaw, S., \& Dowling, N. A. (2018). Therapist-delivered and self-help interventions for gambling problems: A review of contents. Journal of Behavioral Addictions, 7(2), 211-226. doi:10.1556/ 2006.7.2018.44

SBU. (2016). Spel om pengar behandling med psykologiska metoder eller läkemedel vid beroende eller problemspelande (No. 254/2016). Statens beredning för medicinsk och social utvärdering (SBU). Stockholm.

Schafer, J. L. \& Graham, J. W. (2002). Missing data: Our view of the state of the art. Psychological Methods, 7(2), 147-177. doi:10.1037//1082-989X.7.2.147

Shiffman, S., Stone, A. A., \& Hufford, M. R. (2008). Ecological momentary assessment. Annual Review of Clinical Psychology, 4, 1-32. pmid: 18509902

Smith, V. A., Neelon, B., Preisser, J. S., \& Maciejewski, M. L. (2015). A marginalized two-part model for longitudinal semicontinuous data. Statistical Methods in Medical Research. doi: $10.1177 / 0962280215592908$

Toneatto, T. \& Ladoceur, R. (2003). Treatment of pathological gambling: A critical review of the literature. Psychology of Addictive Behaviors: Journal of the Society of Psychologists in Addictive Behaviors, 17(4), 284-292. doi:10.1037/0893-164X.17.4.284. pmid: 14640824

Vehtari, A., Gelman, A., \& Gabry, J. (2017). Practical Bayesian model evaluation using leave-one-out cross-validation and WAIC. Statistics and Computing, 27(5), 1413-1432. doi:10.1007/s11222-016-9696-4

Walker, M., Toneatto, T., Potenza, M. N., Petry, N., Ladouceur, R., Hodgins, D. C., .. Blaszczynski, A. (2006). A framework for reporting outcomes in problem gambling treatment research: The Banff, Alberta Consensus. Addiction, 101 (4), 504-511. doi:10. 1111/j.1360-0443.2005.01341.x

Weinstock, J., Whelan, J. P., \& Meyers, A. W. (2004). Behavioral assessment of gambling: An application of the timeline followback method. Psychological Assessment, 16(1), 72-80. doi:10.1037/1040-3590.16.1.72

Westphal, J. R. (2008). How Well are We Helping Problem Gamblers? An Update to the Evidence Base Supporting Problem Gambling Treatment. International Journal of Mental Health and Addiction, 6(2), 249-264. doi:10.1007/s11469-007-9072-x

Wickwire, E. M., Burke, R. S., Brown, S. A., Parker, J. D., \& May, R. K. (2008). Psychometric evaluation of the National Opinion Research Center DSM-IV Screen for Gambling Problems (NODS). The American Journal on Addictions, 17(5), 392-395. doi:10.1080/10550490802268934. pmid: 18770081

Xing, D., Huang, Y., Chen, H., Zhu, Y., Dagne, G. A., \& Baldwin, J. (2015). Bayesian inference for two-part mixed-effects model using skew distributions, with application to longitudinal semicontinuous alcohol data. Statistical Methods in Medical Research. doi:10.1177/0962280215590284 


\section{Appendix}

Density Function for the Marginalized Two-part Gamma Model

The marginalized two-part model is fit by reparameterizing the classical two-part gamma model. Thus, following Smith et al. (2015), we can write the density for a observation $y$ (omitting the subscripts and random effects) as,

$$
f(y)=\pi \mathbb{1}_{(y=0)} \times(1-\pi) \operatorname{Gamma}(y ; \alpha, \beta) \mathbb{1}_{(y>0)}, \quad y \geq 0,0 \leq \pi \leq 1,
$$

where $\alpha$ is the shape parameter of the gamma distribution, $\beta$ is the rate parameter, $\mathbb{1}$ is the indicator function, and $\pi=\operatorname{Pr}(Y=0)$ gives the probability of $Y$ being zero. In order to estimate the rate parameter we use the marginal mean $(\nu)$ to solve for the conditionally positive mean of the gamma distribution, $\mu=\mathrm{E}(Y \mid Y>0)$. Thus we can write,

$$
\log (\mu)=\log (\nu)-\log (1-\pi) .
$$

Using the fact that the mean of a gamma distribution is $\mu=\frac{\alpha}{\beta}$, gives us the rate parameter,

$$
\beta=\alpha \times \exp (-\mu)
$$

